Supporting Information

\title{
High-Throughput, Algorithmic Determination of Nanoparticle Structure From Electron Microscopy Images
}

Christine R. Laramy, ${ }^{1, \hbar}$ Keith A. Brown, ${ }^{2, \downarrow}$ Matthew N. O’Brien, ${ }^{2}$ and Chad. A. Mirkin ${ }^{1,2^{*}}$

${ }^{1}$ Department of Chemical and Biological Engineering, Northwestern University, Evanston, IL 60208

${ }^{2}$ Department of Chemistry and International Institute for Nanotechnology, Northwestern University, Evanston, IL 60208

'Denotes these authors contributed equally to this work.

Table of Contents:

I. Method of Algorithmic Analysis and Considerations for User Operation 2

a. Summary

b. Image acquisition

c. Code architecture

d. Evaluation of Algorithm Accuracy

II. Additional Characterization of Reaction Class I 9

III. Additional Characterization of Reaction Class II 10

a. Nanoparticle Structural Characterization 10

b. Relative Abundance Study 11

c. Extinction Coefficient Study 12 


\section{METHOD OF ALGORITHMIC ANALYSIS AND CONSIDERATION FOR USER OPEATION}

\section{a. Summary}

BeanCounter.m is the main script responsible for analyzing EM images. Users can pass images into this script through a graphical user interface (GUI) and the script will output a single excel sheet containing particle statistics. If the user selects an entire directory for analysis, BeanCounter.m will aggregate each individual excel sheet into a single excel document. Users can specify the output location of these excel files using the GUI. The excel sheet contains: minor edge length, minor edge length error, aspect ratio, aspect ratio error, corner rounding, corner rounding error, goodness of fit, area, perimeter, and the shape classification or identifier. All errors are determined by the confidence interval of the fit. The shape identifier is a number that corresponds to a particular shape (i.e. 1: rod, 2: circle, 3: triangle, 4: square, 6: hexagon). The GUI has additional options for specifying output images and processing parameters. The following discussion describes additional sample preparation considerations and processing parameters in greater detail.

\section{b. Image Acquisition}

To realize accurate algorithmic nanoparticle detection and analysis, several considerations must be taken during sample preparation and EM image collection.

First, the MATLAB Image Analysis Toolbox is used to initially process images and extract nanoparticle perimeter data. Included in the toolbox is an edge detection function that identifies edges as a gradient between adjacent pixels. When a region is fully contained by edge pixels it is considered a closed object. The custom algorithm analyzes only closed objects as particles. Therefore, the full edge of the nanoparticle must be visible (i.e. not overlapping or fused with adjacent particles) in images. In this way, images with overlapping or fused particles can lead to the analysis of multiple particles as a single particle or prevent particles from being identified as a closed shape (Figure S1). This methodology leads to two primary requirements in the sample preparation and image acquisition process:

1) Only shapes that do not overlap in EM images can be analyzed. For example, octahedron nanoparticles most frequently dry in an orientation with a facet parallel to the substrate. When packed closely, this leads to an overlap of adjacent particles, such that there is no visible border between particles, and therefore this analysis would not work for this particular sample. Another example would be if closely packed cube nanoparticles were imaged at a non-normal angle to their facet, such that particles appeared to overlap in EM images. In this case, the sample can simply be rotated to an angle where the gaps between particles become visible. 
2) Samples must not be fused in a manner that distorts the original nanoparticle shape. Fusion or aggregation, as described here, frequently occurs as a result of sample preparation and can result in two or more distinct particles forming a single shape that is recognized by the analysis. Ideally, this problem can be avoided through proper sample preparation. However, if this cannot be avoided, and the resultant fused nanoparticle shape is not properly fit as one of the primitive shapes selected for analysis, the fused particle can be excluded in the analysis. Alternatively, users can correlate the image analyzed with the associated data file and remove any outliers.
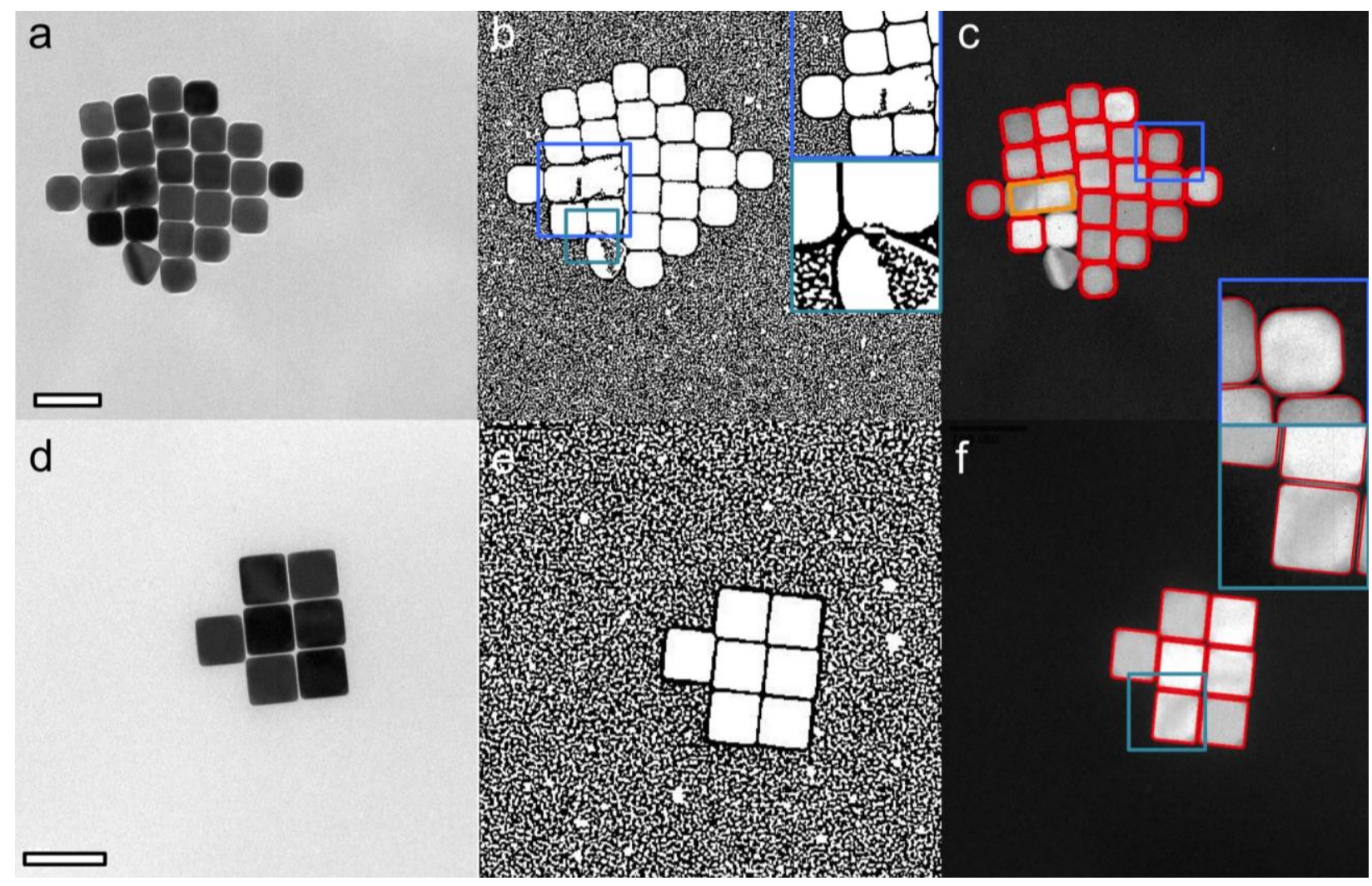

Figure S1. Image processing and analysis steps for an EM image with overlapping and fused particles in comparison to an EM image with fully separate particles. Scale bars represent 100 nm. a) Original TEM image with overlapping and fused particles. b) MATLAB edge detection output for a). Dark blue inset shows fused particles; light blue inset shows overlapping particles. c) Fitting analysis output for a), where fused and overlapping particles are not recognized as cubes (cube recognition denoted by red border). d) Original TEM image with fully separate particles. e) Matlab edge detection output for d), f) Analysis output for e), where light blue inset shows a zoomed in image of fully separated particles. 
Second, the magnification and resolution of the image influences the image processing and the speed of processing. MATLAB's edge detection output may identify closed objects that are not nanoparticles as a result of background noise. These closed objects can be removed from processing by setting thresholds for: the size of closed shapes (in pixels), the solidity of closed shapes, and the average pixel intensity of closed shapes (from the original image).

1) Thresholding the size of closed objects for positive nanoparticle identification can remove background noise (such as the noise in Figure S1b and S1e). More than either of the two additional thresholding steps, size thresholding increases the processing speed of the algorithm. Determining a reasonable number of pixels for area thresholding before processing large amounts of data can save considerable time. Magnification and resolution both influence the number of pixels defining a single nanoparticle by changing the total area the particle takes up within a frame as well as the pixel density within the particle.

2) Thresholding by the average pixel intensity of the closed shape in the original image and solidity removes larger background noise closed objects. These thresholds can become particularly significant for low magnification images.

3) Solidity is the ratio of the area of the closed shape divided by the convex area of the closed shape (with a value ranging from 0 to 1 ). The convex area of an object is the area of the smallest convex polygon (i.e. a polygon with all interior angles less than 180 degrees) that contains all points on the perimeter of the object. Figure S2a below shows a particle with a solidity near 1. Conversely, Figure S2b shows a background object with low solidity.

Together, these three parameters increase speed and improve algorithm accuracy by eliminating non-nanoparticle objects from further processing. (Area, average intensity and solidity thresholds can be modified within the BeanCounter.m file just after image processing steps)

Third, the accuracy of the output of this analysis is limited by the pixilation of the source image. While this may not be significant in the determination of the overall particle size, it may limit the degree to which their corner radius can be computed. As such, the upper bound of this systematic pixilation error is estimated to be equal to the width of a single pixel in EM. Due to this restriction, there is a tradeoff between throughput, resolution, and processing speed that requires optimization depending on experimental conditions. For example, the yield quantification studies described here required a significantly high throughput analysis enabled by lower magnification imaging (i.e. 8,000x). In order to compensate for lower magnification, image resolution was increased (i.e. $2048 \times 2048$ pixels) such that the error was $\leq 0.85 \mathrm{~nm}$. 


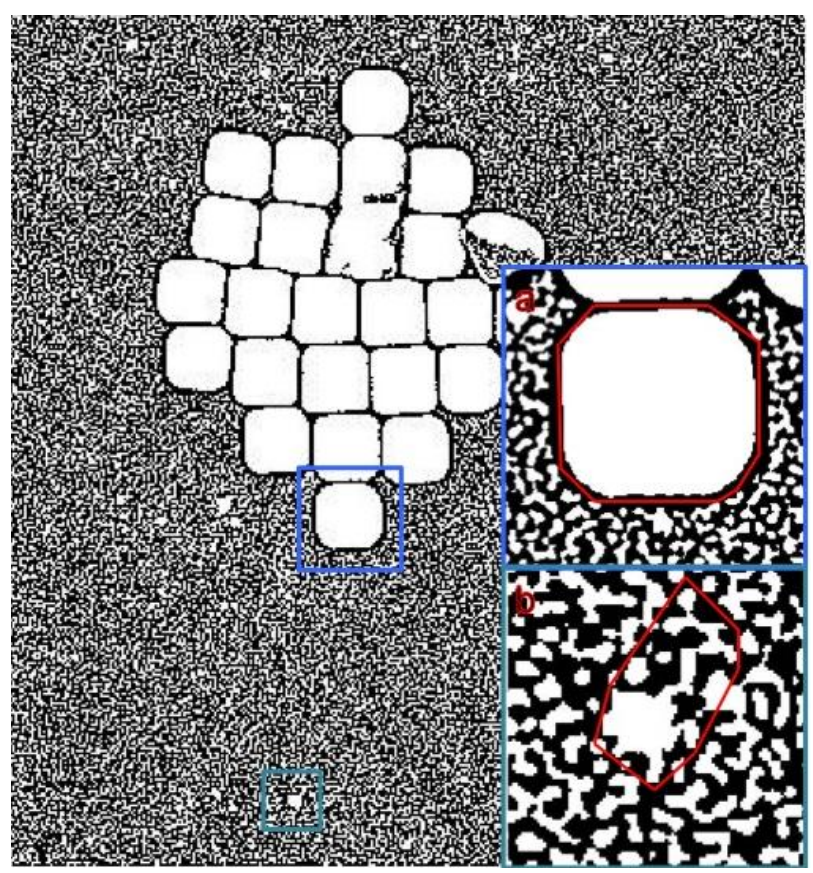

Figure S2. Illustration of convex area determination for solidity calculation. a) convex area is near object area (solidity $\sim 1$ ), b) convex area is greater than object area (solidity $<<1)$

\section{c. Code Architecture}

Once the user inputs "AnalysisGUI" into the MATLAB command window, a GUI appears as shown in Figure S3. From this window the user can select either an image or a directory of images to analyze and a location to save resulting output statistics, along with several other processing parameters including:

- Aspect ratio thresh: This value defines the aspect ratio threshold for classifying a circle or square as a rod. The default setting is 1.3 .

- Pixel conversion factor: This value converts between pixels and physical units. Many image processing software programs have measurement tools available that allow measurement of the pixel length of image scale bars. This value should go here in the format length/pixel.

- Select shapes to fit: These boxes indicate which 2D shapes the program should attempt to fit particles. All may be selected. 
- Select outputs: "Fit Overlay" outputs images such as Figure S1c and S1f for each image. "Minor vs. AR" outputs a plot of minor axis length vs. aspect ratio for all nanoparticles within a single image. "Minor vs. Rounding" produces a similar plot but with corner rounding (r) instead of aspect ratio. "Image Processing" outputs a figure with three images including the original image, an edge detection image, and an image with the closed objects from edge detection filled in (Figure S1b and S1e). Lastly, "Percent of Shapes" outputs a figure with a bar graph representing the percent of each shape fit in the image.

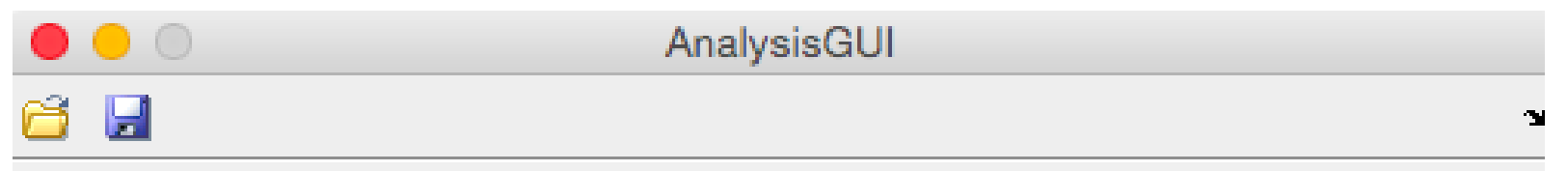

Shape Image Analysis Tool

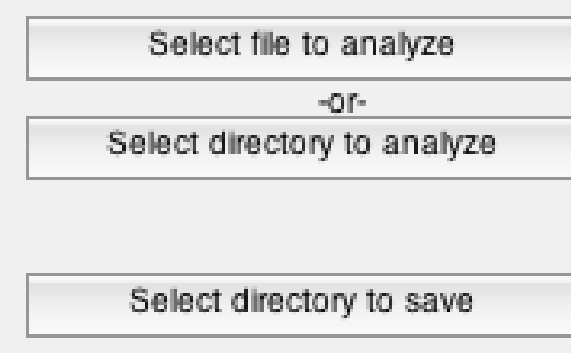

Aspect ratio thresh:

Select shapes to fit

Select outputs

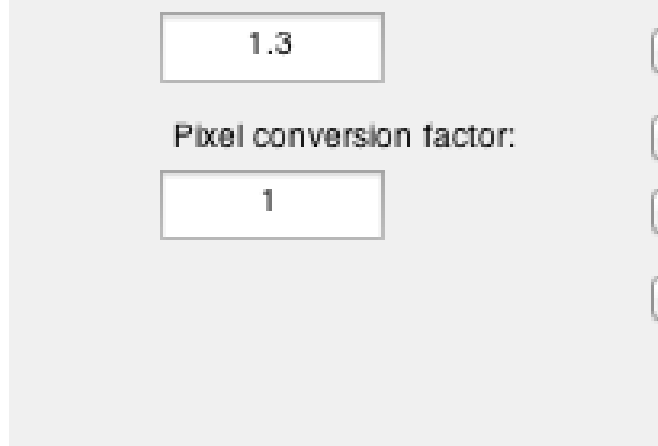

Ellipse

Rectangle

Trlangle

Data

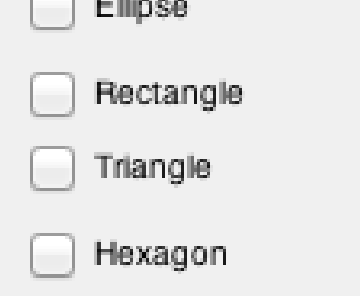

Save

Analyze images

Figure S3. GUI for user input of EM image, output save location, additional processing parameters, and optional output figures

Once the user clicks "Analyze Images" the specified EM image or first image in the specified directory is loaded into BeanCounter.m. A series of image processing and analysis steps, pictorially represented by Figure S4, follow. These include: 
- $\quad$ MATLAB Image Analysis Toolbox image processing. First, BeanCounter.m processes the image and creates a list of pixel coordinates corresponding to the perimeter and centroid of each positively identified particle (filtered by area, intensity and solidity thresholds described previously). The centroid is the coordinate within a closed shape defined as the average of all coordinates (i.e. the center of mass).

- Calculation of $d(\theta)$. BeanCounter.m iterates through the perimeter coordinates of the first particle creating $\mathrm{d}(\theta)$ and $\theta$, a list of the distance and angle between the centroid and the perimeter coordinate.

- Determination of shape and structural parameters. BeanCounter.m passes $\mathrm{d}(\theta)$ and $\theta$ to GetFormics.m which calculates seeds, or starting points, for structural fitting parameters $\left(\mathrm{L}_{\text {major }}, \mathrm{L}_{\text {minor }}\right.$, $\mathrm{r}$, etc.) based on known geometric relationships (i.e. for a circle $d(\theta)$ is equal to the radius and therefore $1 / 2$ of $L_{\text {minor }}$ ). This accelerates nonlinear least squares fitting which requires starting points for fit parameter values. GetFormics.m calculates different seeds for each of four custom functions (MirkEllipse.m, MirkTriangle.m, MirkSquare.m, and MirkHex.m), which together define our library of primitive shapes (circle, triangle, rectangle, and hexagon). GetFormics.m then utilizes native MATLAB fitting tools in the curve fitting toolbox to compute fits to each shape using the associated seeds and $d(\theta)$. The fit with the highest goodness of fit (determined through a nonlinear least squares method) is selected as the best fit. The GetFormics subroutine then returns the best fit to BeanCounter.m.

- Additional structural considerations. BeanCounter.m iterates through each particle collecting fit parameters returned from GetFormics.m. Fit values are converted from pixels to a real space length distance and a final evaluation of which shape to classify each particle is performed. While most particle shapes are determined by the fit shape returned by GetFormics.m, BeanCounter.m reclassifies some particles as rods by comparing the aspect ratio of fit parameters returned for circle and square fits against the user set aspect ratio threshold. Additionally, BeanCounter.m will reclassify highly rounded shapes as circles (i.e. as $\mathrm{r}$ approaches a fractional value of $\mathrm{L}$, this threshold can be modified in BeanCounter.m).

- Data output. Lastly, BeanCounter.m aggregates final particle statistics and outputs a single excel file for each EM image. If a whole directory is selected, an excel sheet containing the total statistics for each image in the directory is output. An example output can be seen in Figure S4. In addition to an excel sheet containing all population statistics, a second excel sheet is produced that contains the total nanoparticle shape yield within the analyzed population. 


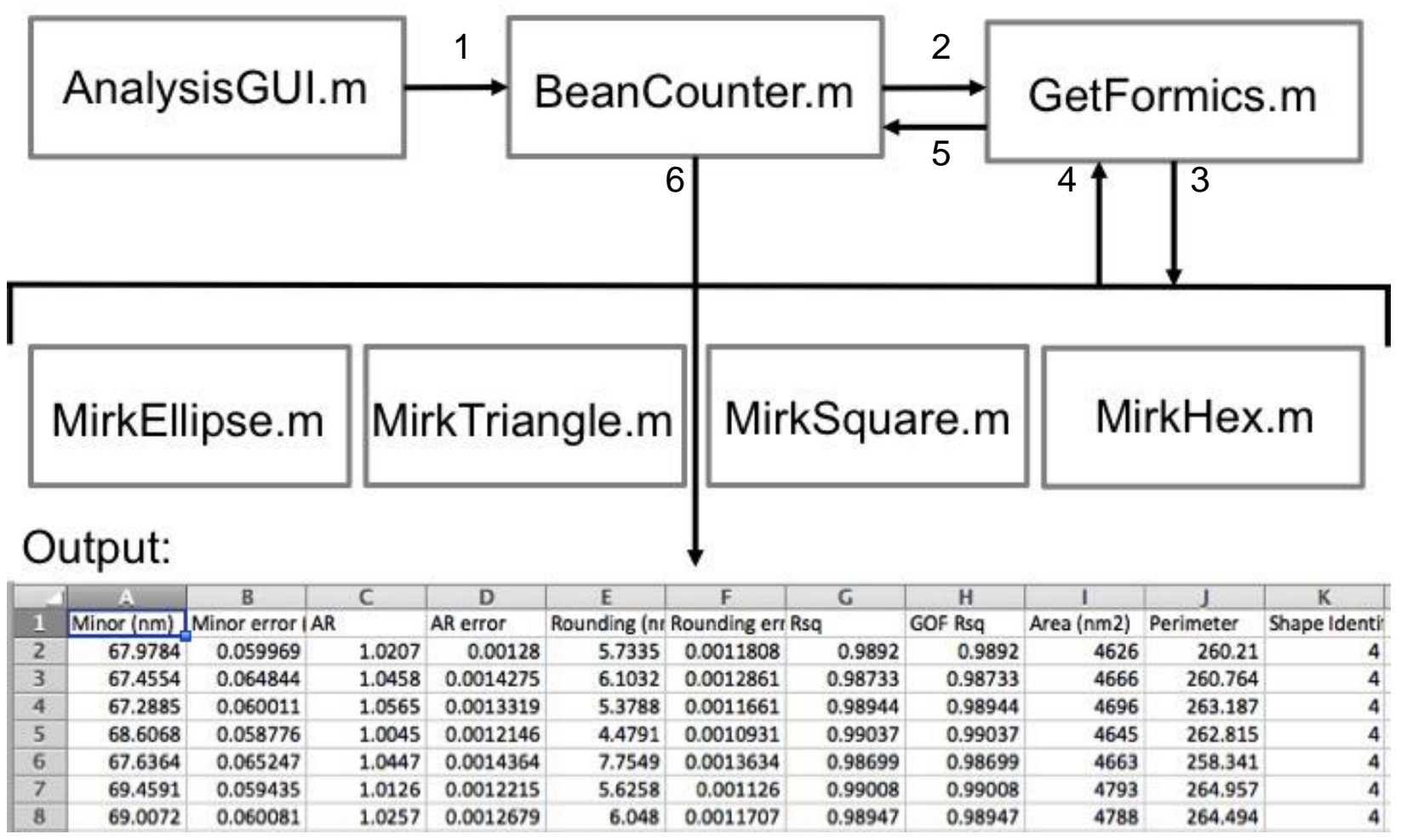

Figure S4. EM image processing and analysis architecture and example output, numbers indicate processing order. 


\section{d. Evaluation of Algorithm Measurement Accuracy}

In order to evaluate the accuracy of our algorithmic image analysis software we compared the data from the algorithm with data calculated manually using a measurement tool common in image editing software (specifically, the "Measurement" tool in Adobe Photoshop). Two authors separately measured $\mathrm{L}_{\text {minor }}$ and $\mathrm{L}_{\text {major }}$ (and thus calculated an AR) from EM images for $\sim 150$ cubes. Figure S5 shows both manual calculations as well as the computational output. The computational data shows strong agreement with both sets of manual data.

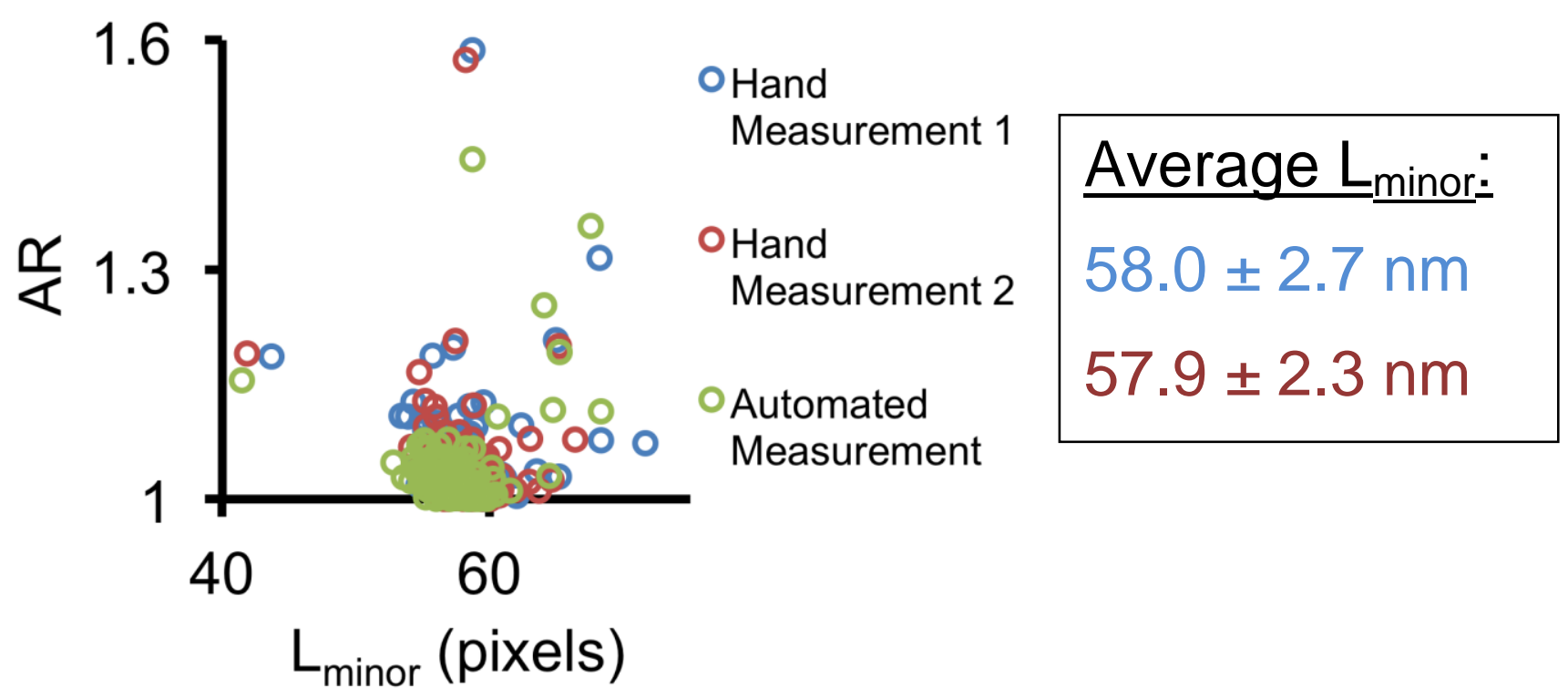

Figure S5. A comparison of computationally measured particle structure vs. manual measurements using Adobe Photoshop. 


\section{Additional Characterization for Reaction Class I}

Figure S6 below shows representative EM images at each time point during triangular prism dissolution.

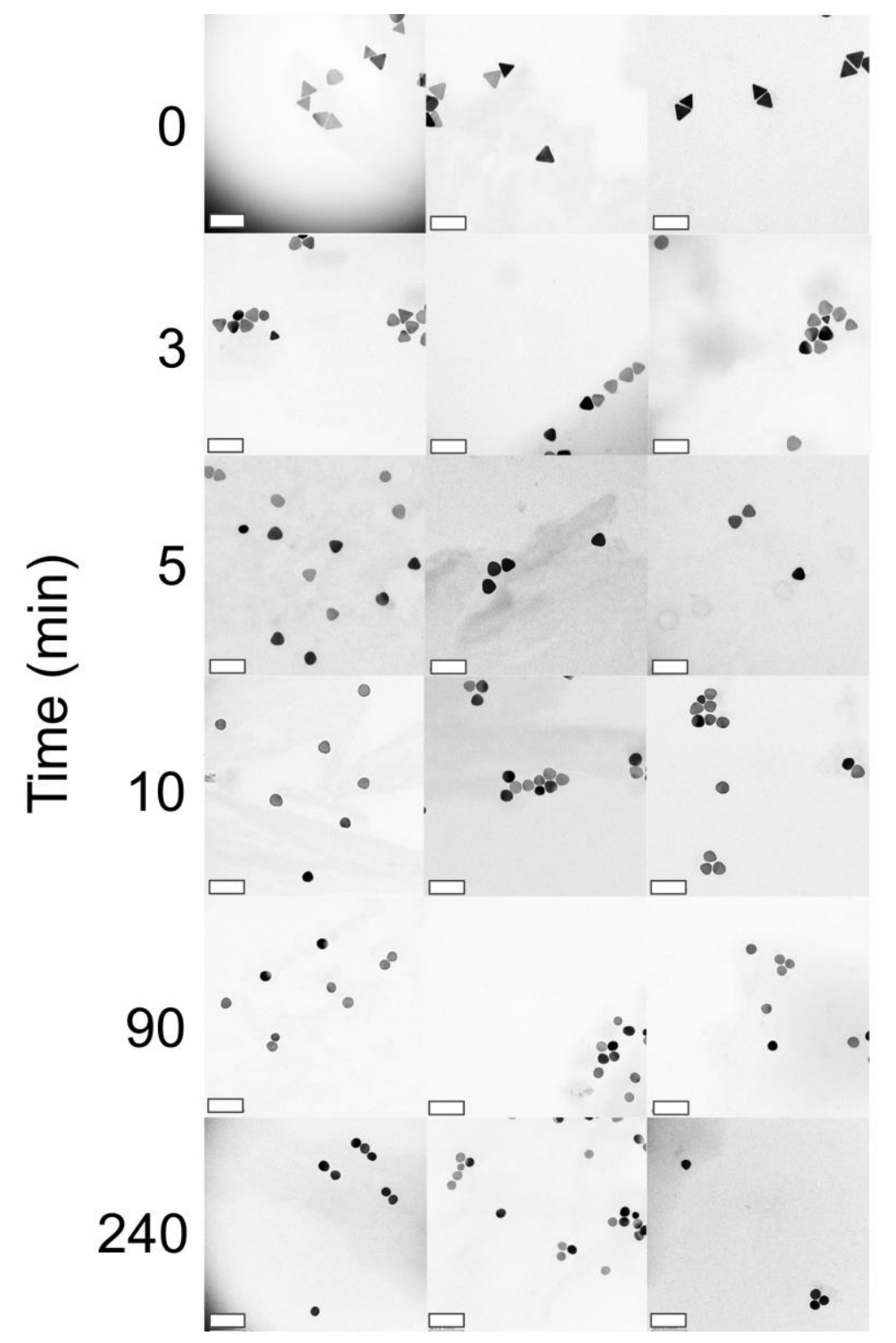

Figure S6. Three representative EM Images for each time point during prism dissolution. Scale bars represent $200 \mathrm{~nm}$. 


\section{Additional Characterization of Reaction Class II}

\section{a. Nanoparticle Structural Characterization}

Before use in both the relative abundance study and the extinction coefficient study, we characterized the structure of spheres, cubes and rods in order to accurately determine an extinction coefficient based on reported values. ${ }^{1,2}$ Figure S7 shows representative EM images, UV-Vis extinction spectra, and population statistics from our automated analysis of EM images. Table S1 lists the extinction coefficients chosen for each particle species.
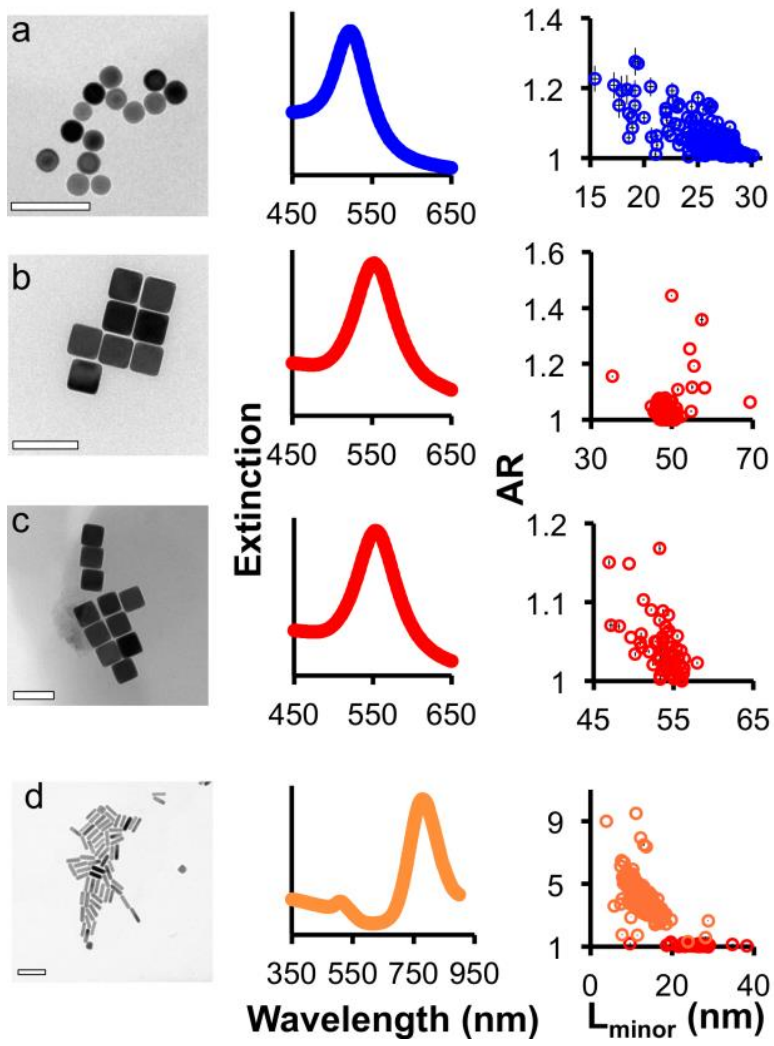

Figure S7. Structural characterization of particle species for both the relative abundance and rod extinction coefficient studies by UV-Vis spectroscopy and algorithmic EM image analysis. Color scheme is consistent with Figure $3 b$ for distinguishing sphere vs. cube vs. rod data. Each row shows a representative EM image, UV-Vis spectroscopy measurement, and algorithmic EM image analysis for a particle species. All scale bars represent $100 \mathrm{~nm}$. a) Characterization of spheres for both the relative abundance study and the rod extinction coefficient study, b) Characterization of cubes for the relative abundance study, c) Characterization of cubes for the rod extinction coefficient study, d) Characterization of rods for the rod extinction coefficient study. Red data points are consistent with the color scheme and indicate cube impurities from rod synthesis. 
Table S1. $\varepsilon$ for the species used in this study

\begin{tabular}{|l|l|}
\hline Species & $\boldsymbol{\varepsilon}\left(\mathbf{M}^{-1} \mathbf{c m}^{-1}\right)$ \\
\hline Sphere $^{3}$ & $4.0 \times 10^{9}$ \\
\hline Cube (Figure S3b) & $1.0 \times 10^{11}$ \\
\hline Cube (Figure S3c) & $1.0 \times 10^{11}$ \\
\hline
\end{tabular}

\section{b. Relative Abundance Study}

Concentrated stock solutions of cubes and spheres (in $50 \mathrm{mM}$ CTAB) were each measured with UV-Vis following dilution with water, and concentrations were determined using Table S1. A $750 \mu \mathrm{L}$ solution was then prepared containing cubes and spheres at $40 \mathrm{pM}$, diluted with water. This solution was then centrifuged to remove CTAB (5283 rcf, 11min) and particles were re-suspended in $750 \mu \mathrm{L}$ of nanopure water. Next, $9 \mu \mathrm{L}$ of the nanoparticle solution was drop cast on a copper TEM grid and immediately wicked away using filter paper to reduce evaporative drying effects. This drop casting and wicking process was repeated 10 times. Grids were prepared in triplicate using the same solution and preparation procedure. The copper grids were imaged immediately following sample preparation. Representative images for each of the three samples are shown below in Figure S8. Analysis of these samples by EM shows that spheres and cubes were found in a 1:1 ratio within experimental error (Figure S9).

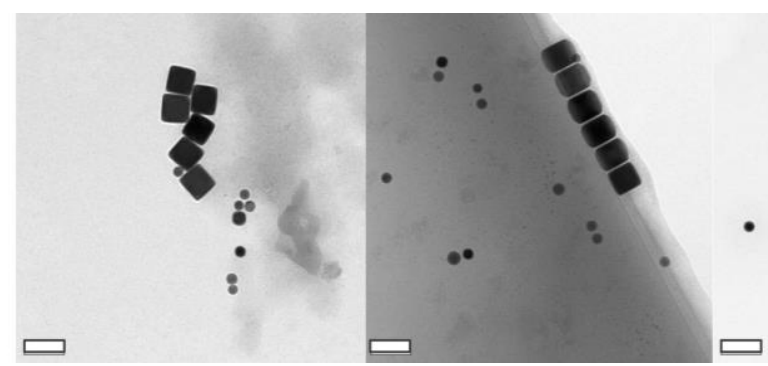

Figure S8. Representative EM images for the relative abundance study. Scale bars represent $100 \mathrm{~nm}$. 


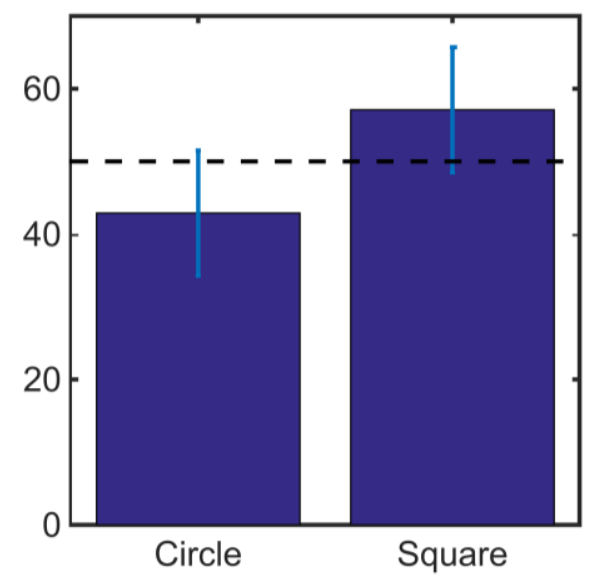

Figure S9. Relative amounts of Spheres (Circle) and Cubes (Square) calculated with automated analysis from EM images. The dashed line indicates 50\% (the expected relative amount of both spheres and cubes). Error bars represent the standard deviation of three samples.

\section{c. Extinction Coefficient Study}

Figure S10 below shows representative EM images for each of the three experimental conditions investigated. In each of these conditions, a different $\varepsilon$ was used to calculate the concentration of rods and to set an expected ratio of cube:sphere:rod of 1:4:8, respectively. Exact $\varepsilon$ values and their sources are listed in the caption of Figure 3. 


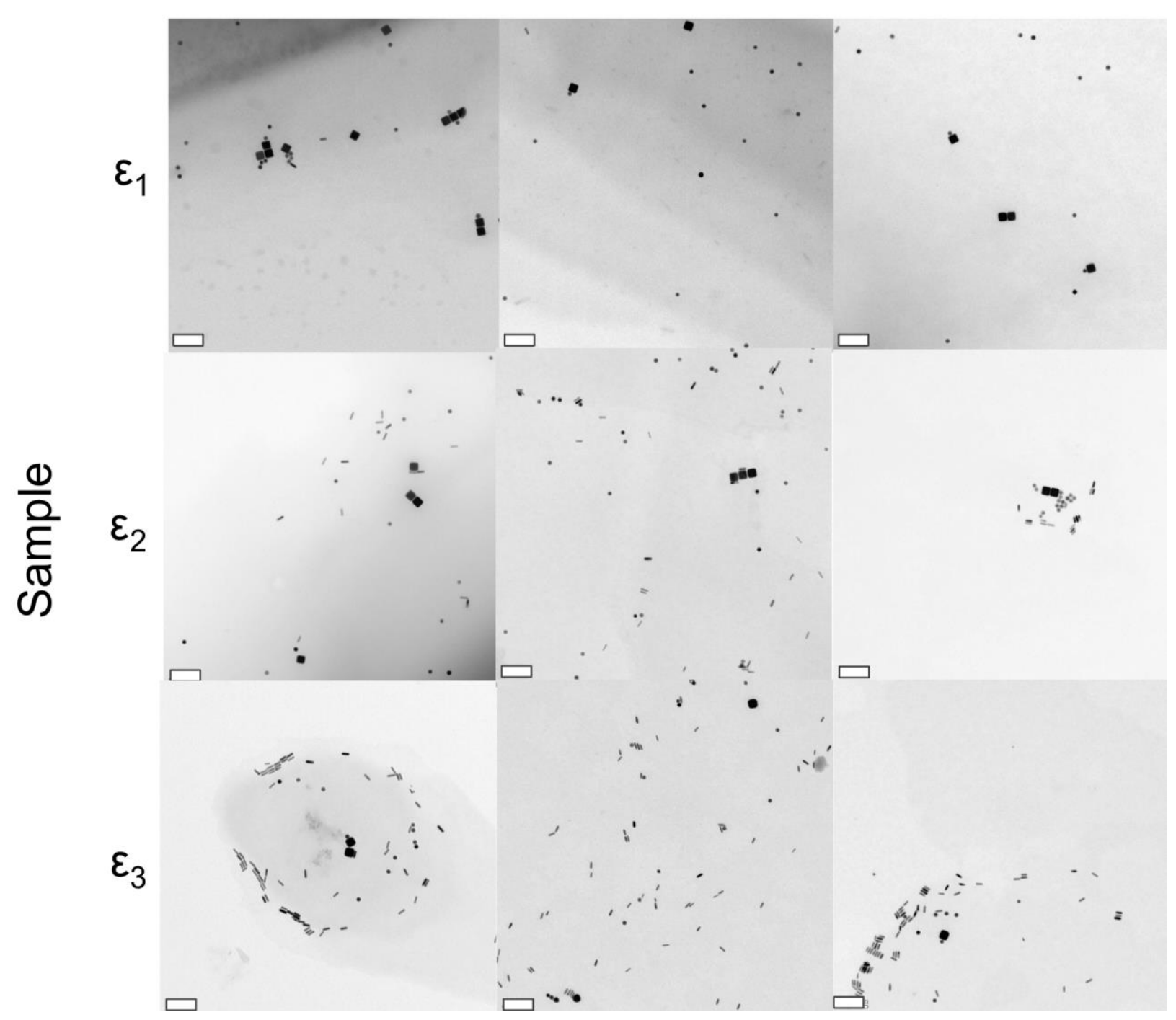

Figure S10. Representative EM images from each of the three experimental conditions investigated in Figure 3. Scale bars represent $200 \mathrm{~nm}$.

Using the nanoparticle density and magnifications described above, we find an average of 40 nanoparticles per frame. As the image does not need to be magnified, focused, or contrastadjusted between frames, we find that imaging the 25 frames needed to accumulate 1000 nanoparticles took $\sim 30$ minutes after the initial focusing process. Computational image analysis of these 25 images requires less than 10 minutes. In total the process of analyzing a sample requires less than one hour. 


\section{References:}

1. O'Brien, M. N.; Jones, M. R.; Brown, K. A.; Mirkin, C. A. Universal Noble Metal Nanoparticle Seeds Realized through Iterative Reductive Growth and Oxidative Dissolution Reactions. J. Am. Chem. Soc 2014, 136, 7603-7606.

2. Extinction coefficient obtained from product information provided by Ted Pella, Inc.

3. Extinction coefficient obtained from product information provided by Ted Pella, Inc. 